\title{
Rotatorenmanschettenruptur: Wann ist die konservative und wann die operative Therapie indiziert?
}

\author{
Wieser, Karl ; Bouaicha, Samy ; Grubhofer, Florian
}

\begin{abstract}
Rotator Cuff Rupture: When Is Conservative and When Is Surgical Therapy Indicated? Abstract. Patients with rotator cuff tears can be treated conservatively or surgically. The treatment decision depends on the one hand on patient-specific factors like shoulder impairment, age, activity level, functional demand, and on the other hand on morphological or radiological circumstances like tear size, muscle degeneration and fatty infiltration of the rotator cuff muscles. In this review, the treatment approach of our institution is described.
\end{abstract}

DOI: https://doi.org/10.1024/1661-8157/a003193

Other titles: Rotator Cuff Rupture: When Is Conservative and When Is Surgical Therapy Indicated?

Posted at the Zurich Open Repository and Archive, University of Zurich

ZORA URL: https://doi.org/10.5167/uzh-170094

Journal Article

Published Version

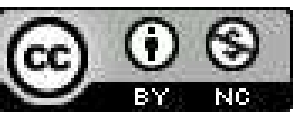

The following work is licensed under a Creative Commons: Attribution-NonCommercial 3.0 Unported (CC BY-NC 3.0) License.

Originally published at:

Wieser, Karl; Bouaicha, Samy; Grubhofer, Florian (2019). Rotatorenmanschettenruptur: Wann ist die konservative und wann die operative Therapie indiziert? Praxis, 108(4):257-268.

DOI: https://doi.org/10.1024/1661-8157/a003193 


\title{
Rotatorenmanschettenruptur:
}

\section{Wann ist die konservative und wann} die operative Therapie indiziert?

\author{
Rotator Cuff Rupture: When Is Conservative and When Is \\ Surgical Therapy Indicated?
}

Karl Wieser, Samy Bouaicha und Florian Grubhofer

Universitätsklinik Balgrist, Zürich

\begin{abstract}
Zusammenfassung: Patienten mit Rotatorenmanschettenrupturen können konservativ oder operativ behandelt werden. Welche Behandlung gewählt wird, hängt einerseits von patientenspezifischen Faktoren wie Leidensdruck, körperliche Einschränkung, Aktivitätsniveau und Funktionsanspruch ab und andererseits von morphologischen/radiologischen Gegebenheiten wie Grösse der Sehnenruptur, Degeneration und Verfettung der Rotatorenmanschettenmuskulatur sowie Begleitverletzungen. Dieser Artikel beleuchtet die Hintergründe der Indikationsstellung für die konservative und operative Behandlung von Patienten mit Rissen der Rotatorenmanschette unter Einbezug klinischer und radiologischer Kriterien.
\end{abstract}

Schlüsselwörter: Rotatorenmanschettenruptur, konservative Therapie, operative Therapie, Indikation zur Operation bei Rotatorenmanschettenruptur

\begin{abstract}
Patients with rotator cuff tears can be treated conservatively or surgically. The treatment decision depends on the one hand on patient-specific factors like shoulder impairment, age, activity level, functional demand, and on the other hand on morphological or radiological circumstances like tear size, muscle degeneration and fatty infiltration of the rotator cuff muscles. In this review, the treatment approach of our institution is described.
\end{abstract}

Keywords: Rotator cuff tear, conservative versus surgical treatment, treatment approach for rotator cuff tear

\begin{abstract}
Résumé: Les patients présentant des ruptures de coiffe des rotateurs peuvent être traités de façon conservatrice ou chirurgicale. Le choix du traitement dépend d'une part de facteurs spécifiques au patient tels que la souffrance, les limitations physiques, le niveau d'activité et les exigences fonctionnelles, et d'autre part de conditions morphologiques/radiologiques telles que la taille de la rupture du tendon, la dégénérescence et la dégénérescence graisseuse des muscles de la coiffe des rotateurs ainsi que des blessures qui les accompagnent. Cet article met en lumière le contexte de l'indication pour le traitement conservateur et chirurgical des patients présentant des déchirures de la coiffe des rotateurs, y compris les critères cliniques et radiologiques.
\end{abstract}

Mots-clés: Rupture de la coiffe du rotateur, thérapie conservatrice, thérapie chirurgicale, indication d'une chirurgie pour rupture de la coiffe des rotateurs

Risse der Rotatorenmanschette (RM) sind eine vor allem mit zunehmendem Patientenalter durchaus häufige Pathologie des Schultergelenks und sind selbst bei asymptomatischen, über 60-jährigen Menschen in gut einem Drittel und bei asymptomatischen über 80-Jährigen in über $50 \%$ der Fälle nachweisbar [1, 2]. Dieses Wissen deutet darauf hin, dass der alleinige radiologische Nachweis einer vollständigen Ruptur der RM noch lange keinen operativen Eingriff per se benötigt. Aber nicht nur der allgemein praktizierende Arzt, sondern auch der Patient und nicht selten auch der Schulterspezialist stehen immer wieder vor der schwierigen Entscheidung, ob die jeweilige RM-Ruptur nun konservativ oder operativ behandelt werden soll. Das übergeordnete Ziel jeglicher orthopädischer Behandlung wie auch der Therapie von RM-Läsionen ist es, Patienten wieder an ihr gewohntes Aktivitätsniveau heranzuführen

$\begin{array}{ll}\text { Im Artikel verwendete Abkürzungen } \\ \text { AC-Gelenk } & \text { Acromioclaviculargelenk } \\ \text { MRI } & \text { Magnetresonanztomografie } \\ \text { RM } & \text { Rotatorenmanschette } \\ \text { RMR } & \text { Rotatorenmanschettenrekonstruktion } \\ \text { SAS } & \text { Schulterarthroskopie }\end{array}$

\section{Im Artikel verwendete Abkürzungen}

AC-Gelenk Acromioclaviculargelenk

RM Rotatorenmanschette

SAS Schulterarthroskopie 
und wenn möglich einer zukünftigen Verschlechterung der Situation vorzubeugen. Auch wenn einzelne Fachgesellschaften eigene Behandlungsalgorithmen empfehlen [35], gibt es keinen international anerkannten Standard für die Behandlung von Rotatorenmanschettenrupturen, und dementsprechend variieren die Vorgehensweisen innerhalb der Schulterchirurgie stark [6]. Die teils sehr hohen Rerupturraten nach Rotatorenmanschettenrekonstruktion (RMR) von bis zu 20-30\% [7, 8] sind meist auf schwierige biologische und biomechanische Grundvoraussetzungen (Rupturgrösse und Sehnen-/Muskeldegeneration) zurückzuführen. Wird eine RMR bei einer stark verfetten RMMuskulatur und bei entsprechend stark retrahierter Sehne durchgeführt, sind sogar Versagensraten von $>90 \% \mathrm{zu}$ beobachten $[9,10]$. Aufgrund dieser Beobachtungen werden diese RM-Rupturen als irreparabel bezeichnet und sollten nicht mehr mit einer direkten RMR behandelt werden.

In diesem Artikel beleuchten wir die Hintergründe, wie an unserer Klinik entschieden wird, wie ein Patient mit einer RM-Ruptur behandelt wird.

Die wichtigsten Parameter, die für den Therapieentscheid notwendig sind und mittels Anamnese, klinischer und radiologischer Untersuchung erhoben werden, sind:

1. Schmerzen

2. Schulterfunktion (aktiver Bewegungsumfang, Schwäche)

3. Aktivitätsniveau des Patienten

4. Grösse der Sehnenruptur (MRI: Partial-/Transmural-/ Massenruptur)

5. Sehnen und Muskelqualität (MRI: Sehnenretraktion, Muskeldegeneration)

\section{Anamnese}

Die Erfassung der Schmerzdauer (Wochen, Monate, Jahre), der Intensität (visuelle analoge Skala 0-10) und des subjektiven Schulterwertes [11] helfen dem Untersucher, einen Eindruck vom Leidensdruck des Patienten zu erhalten. Die Schmerzlokalisation und die Schmerzausstrahlung sind zu erfragen, da Schulterschmerzen auch als Symptom von Pathologien aus benachbarten Gelenksregionen wie der Halswirbelsäule (radikuläre Schmerzsymptomatik mit oder ohne sensomotorische Defizite) oder dem Acromioclaviculargelenk (AC) (Schmerzmaximum bei Palpation des AC-Gelenks) auftreten können. Der für die RM-Ruptur typische Schmerz strahlt in die proximale laterale und/oder ventrale Oberarmregion aus und stört die Patienten häufig auch während der Nachtruhe. Wichtig ist die anamnestische Differenzierung zwischen Schmerzen und Schwäche. Schmerzen allein sind häufig konservativ erfolgreich zu behandeln. Die Schwäche stellt jedoch in der Regel ein funktionelles Problem dar und ist durch eine konservative Therapie selten zu verbessern. Es gibt keine suffiziente synergistische Muskulatur, die z.B. eine kombinierte Supra- und Infraspinatusruptur die Elevations- und Aussenrotationsschwäche kompensieren kann. Während Schmerzen für den Patienten üblicherweise im Vordergrund stehen und im Anamnesegespräch entsprechend vom Patienten genannt werden [12], ist eine Schwäche der Schulter weniger häufig Thema und muss daher vom Untersucher aktiv erfragt und gezielt untersucht werden. Seitliche und vordere Schulterschmerzen können jedoch auch Ausdruck einer subacromialen Bursitis, einer Capsulitis adhaesiva (Frozen Shoulder), oder einer Omarthrose sein. Die Differenzierung gelingt durch die sorgfältige klinische Untersuchung und weitergehende bildgebende Abklärungen (s.u.). Traumatische Rupturen sind im Vergleich zu den viel häufiger auftretenden degenerativen Rupturen [13] mit einem adäquaten Unfallereignis verbunden. Kann ein adäquates auslösendes Schultertrauma mit anschliessender Schwäche für Über-Kopf- oder Rotationsbewegungen erhoben werden, sollte die Schulteruntersuchung inkl. Bildgebung (s.u.) zeitnahe erfolgen, da insbesondere Patienten mit hohem Funktionsanspruch deutlich von einer frühzeitigen chirurgischen Rekonstruktion der RM profitieren können [14].

Insgesamt sind für die weitere Abklärung und $\mathrm{Be}-$ handlung der allgemeine Gesundheitszustand und das Aktivitätsniveau des Patienten ausschlaggebend. Dementsprechend sollten in der Arbeitsanamnese das körperliche Belastungsprofil am Arbeitsplatz und in der Freizeitanamnese Hobbies, sportliche Tätigkeiten, Gartenarbeiten, Hausarbeiten, etc. erhoben werden. Aktive Patienten bzw. körperlich berufstätige Patienten sind auf eine mechanisch intakte RM deutlich häufiger angewiesen als Patienten mit niedrigem Beanspruchungsprofil. Diese können trotz partieller oder gelegentlich auch transmuraler RM-Ruptur gute und lang anhaltende Behandlungsergebnisse mit konservativer Therapie aufweisen $[15,16]$.

\section{Klinische Untersuchung}

Mit Hilfe der klinischen Untersuchung soll einerseits die anamnestische Verdachtsdiagnose untermauert und die Grundlage für weitergehende bildgebende Abklärungen gelegt werden. Jene Untersuchungsbefunde, die ein proaktives Handeln erfordern, werden in diesem Abschnitt hervorgehoben.

\section{Inspektion}

Bei der Inspektion wird neben Operationsnarben, vor allem auf eine eventuell vorhandene Muskelatrophie im Bereich von Fossa supra- und infraspinata geachtet. Sowohl die eventuelle Muskelatrophie als auch eine weit seltener vorkommende sichtbare anterosuperiore Subluxation des Humeruskopfs sind mit einer chronischen RMRuptur assoziiert, die entweder i.d.R. als irreparabel einzustufen oder prognostisch mit einer sehr hohen Versagensrate der RMR verbunden sind 17-19]. 


\section{Passive Beweglichkeit}

Die passive Beweglichkeit des glenohumeralen Gelenks wird im Seitenvergleich geprüft. Eine erhöhte Aussenrotationsamplitude gilt als pathognomonisch für eine Subscapularisläsion [20]. Zeigt die erkrankte oder verletzte Schulter eine im Seitenvergleich verminderte glenohumerale Beweglichkeit (v.a. Aussenrotationsfähigkeit), ist dies meist Ausdruck einer entzündlichen glenohumeralen Kapselschrumpfung (Frozen Shoulder/Capsulitis adhaesiva).

\section{Aktive Beweglichkeit}

Bei der aktiven Bewegungsprüfung der Flexion/Extension, Aussenrotation/Innenrotation, sowie Abduktion/Adduktion wird auf das Vorhandensein einer sogenannten Pseudoparalyse geachtet. Die pseudoparalytische Schulter kann passiv uneingeschränkt bewegt werden, aktiv kann der Patient seinen Arm jedoch nicht auf und über $90^{\circ}$ anheben bzw. dort halten [21]. Dies ist, bei Ausschluss einer neurologischen Schädigung (echte Paralyse) pathognomisch für eine posterosuperiore Massenruptur der RM. Kann der Patient, bei erhaltener passiver Aussenrotationsfähigkeit mit dem Ellbogen am Rumpf, diese Endposition nicht halten und es kommt zu einem Zurückfedern des Armes um $>15^{\circ}$, so spricht man von einem positiven Aussenrotationslag, was ein Hinweis für eine Infraspinatussehnenruptur ist [22]. Besteht ein Aussenrotationslag auch bei $90^{\circ}$ abduziertem Arm, so ist die Wahrscheinlichkeit für eine kombinierte Infraspinatus- und Teres-minor-Sehnenruptur sehr hoch [23]. Die kombinierte Ruptur der beiden RMSehnen kann sehr gut mit dem «Hand-zu-Mund-Test» entlarvt werden. Wenn ein Patient mit rupturierter posteriorer RM (Infraspinatus- und Teres-minor-Sehne) die Hand zum Mund führen soll, kann er dies nur mit Hilfe von vermehrtem Abduktionseinsatz des intakten Musculus deltoideus und entsprechend forciertem Abspreizen des Ellbogens. Da diese charakteristische Armstellung bzw. Ausweichbewegung auch beim Blasen des Horninstruments eingenommen wird, ist die pathologische Hand-zu-Mundbewegung auch als «Hornblower»-Zeichen bekannt (Abb. 1) [23].

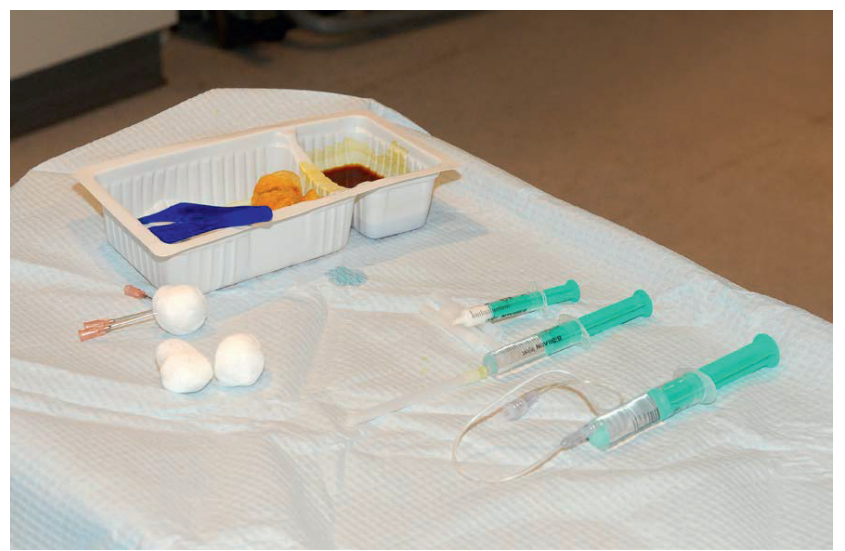

Abbildung 1. Steriles Instrumentarium für die Infiltration am «Back Table».

\section{Rotatorenmanschetten-Funktionstests}

Zur gezielten Überprüfung der RM-Integrität stehen eine Vielzahl an RM-Tests zur Verfügung [24]. Es ist nicht zielführend, jedes Mal alle bekannten Tests durchzuführen. Der Untersucher sollte sich vielmehr auf einen standardisierten Untersuchungsgang mit Einbezug der für ihn wichtigsten Tests beschränken. Grundsätzlich sind die Funktionstests bei Schwäche im Seitenvergleich als positiv zu werten. Schmerzprovokation mit konsekutiver Muskelinhibition darf nicht als positives Testergebnis fehlinterpretiert werden. Die Tests mit der höchsten Spezifität, respektive Sensitivität, werden hier aufgeführt: Der Jobe-Test [25] und Whipple-Test [26] eignen sich für den Nachweis einer Supraspinatussehnenruptur [27]. Der Lift-off-Test [20] und der Belly-press-Test [28] sind beim Vorhandensein einer Subscapularissehneruptur am häufigsten positiv. Die Aussenrotationschwäche in $0^{\circ}$ Abduktion und das «External-Rotation Lag Sign» in $0^{\circ}$ Schulterabduktion [22] sind auf eine Infraspinatussehnenruptur hinweisend. Das «Hornblower»-Zeichen [23, 29] ist - wie bereits oben beschrieben - pathognomisch für die kombinierte Ruptur der Infraspinatus- und Teres-minor-Sehne. Falls die Funktionstests wegen Schmerzen nicht durchgeführt werden können, kann durch die subacromiale Infiltration von Lokalanästhetikum eine Schmerzreduktion erzielt werden, um so die Kraftprüfungen der einzelnen Sehnen zuverlässiger durchführen zu können (Neer-Test) [30].

\section{Radiologische Untersuchungen}

\section{Röntgen}

Die standardmässige durchgeführte Röntgenuntersuchung der Schulter besteht aus drei Aufnahmen in drei verschiedenen Ebenen (anteroposteriore-, axiale- und Neer $(=\mathrm{Y})$ Aufnahme).

Neben radiologischen glenohumeralen Arthrosezeichen (Gelenksspaltverschmälerung, Osteophyten, Zysten) wird insbesondere die Zentrierung des Humeruskopfs beurteilt. Eine radiologische Dezentrierung nach kranial mit Verminderung der acromiohumeralen Distanz (ACHD) von $<7 \mathrm{~mm}$, weist auf eine posterosuperiore (Supraspinatus- und Infraspinatussehnen-)RM-Ruptur hin [31] und ist gleichzeitig prognostisch mit einer hohen Rekonstruktionsversagensrate assoziiert [17, 32]. Dementsprechend ist bei vorhandener Arthrose und/oder proximaler Migration des Humeruskopfs häufig eine gelenkserhaltende Sehnenrekonstruktion oder ein Sehnentransfer nicht mehr sinnvoll.

\section{Ultraschalluntersuchung}

Die Ultraschalluntersuchung kann mit einer Spezifität von $94 \%$ und einer Sensitivität von $92 \%$ gut zur Bestätigung 
und auch zum Ausschluss der Diagnose einer transmuralen RM-Ruptur eingesetzt werden [33]. Für den Ausschluss einer Partialruptur ist die Ultraschalluntersuchung mit einer Sensitivität von $64 \%$ weniger gut geeignet [33]. Entsprechend lässt sich die Ultraschalluntersuchung gut für die postoperative Kontrolle nach einer RMR einsetzen. Die Ultraschalluntersuchung kann zudem die fettige Infiltration der RM-Ruptur, die entscheidend für die Therapieform ist, nicht verlässlich erfassen. Dies ist ein wesentlicher Grund, weshalb die MRI-Untersuchung häufiger zur Abklärung von RM-Rupturen angewandt wird.

\section{Magnetresonanztomografie (MRI)}

Der besondere Stellenwert der MRI-Untersuchung liegt neben dem definitiven Ruptur-Nachweis (inkl. Grösse, Lage und Retraktion des Sehnenrisses [34]) in der Darstellbarkeit der allfällig nach Sehnenriss bereits aufgetretenen sekundären Muskeldegeneration. Sowohl die Grösse der Partial- als auch der Transmuralrupturen kann anhand von verschiedenen Klassifikationen eingeteilt werden [35, 36]. Das Verfettungsausmass kann in der sagittalen Schnittbildaufnahme sowohl im MRI als auch im CT nach Goutallier [37,38] quantifiziert und eingeteilt werden. Sehnenrupturen mit Verfettungsstufen Goutallier 3 und 4 (dies entspricht gleich viel oder mehr Fettgehalt als Muskelanteil) sind ausserordentlich häufig mit hohen Rekonstruktionssversagen verbunden und werden daher als irreparable RM-Rupturen bezeichnet $[38,39]$.

\section{Therapie}

Die Therapie eines Patienten mit einer RM-Ruptur hängt in erster Linie davon ab, ob der Patient einen durch die Ruptur verursachten Leidensdruck verspürt oder nicht. Dies hängt wiederum meist vom Funktionsanspruch und dem Alter (inkl. Komorbiditäten) des Patienten ab.

\section{Konservative Therapie}

Nicht jede symptomatische RM-Ruptur muss operativ behandelt werden. Hausinterne Studien konnten zeigen, dass sowohl Patienten mit Partialrupturen [15] als auch mit Massenrupturen [16] durch konservative Therapiemassnahmen akzeptable Behandlungsergebnisse erreichen können.

Die konservative Therapie hat einerseits das Ziel, die Schmerzen zu reduzieren, und anderseits, die Schulterfunktion zu verbessern.

Die von uns angewandten und auch von der American Academy of Orthopaedic Surgeons anerkannten konservativen Therapiemassnahmen für die Behandlung von RM Rupturen sind [3]:
- Medikamentöse Therapie (NSAR)

- Infiltrationstherapie mit Depotsteroiden (z.B: $40 \mathrm{mg}$ Triamcinolon in $5 \mathrm{ml} 2 \%$ Lidocain)

- Manuelle schulterspezifische Physiotherapie

- Ergotherapie zur Optimierung der «Activities of Daily Living»

- Anpassung des Lebensstils

\section{Infiltration der Schulter}

Bei transmuralen Rupturen kann die Depotsteroidinfiltration in Kombination mit kurzwirksamen Lokalanästhetikum über eine sogenannte subacromiale Infiltration erfolgen, da sich über die transmurale Ruptur das subacromial applizierte Depot sowohl subacromial als auch intraartikulär verteilt.

Die Infiltration soll und darf nur unter höchsten Hygienebedingungen stattfinden. Die Folgen einer iatrogenen septischen Arthritis sind für das Gelenk und die betroffenen Patienten verheerend und gehen typischerweise mit einer invalidisierenden Destruktion des Gelenksknorpels und gelegentlich mit einer konsekutiven, schwer behandelbaren Osteomyelitis einher. Idealerweise wird ein eigens eingerichteter «Punktionsraum» für Infiltrationen verwendet. In diesem Raum müssen vor und während der Punktion die Fenster und Türen geschlossen sein. Vor dem Zimmer soll während der Infiltration darauf verwiesen werden, dass eine Gelenksinfiltration stattfindet, damit die Tür während der Infiltration nicht geöffnet wird. Die anwesenden Personen im Raum sollen auf das Minimum reduziert sein (d.h. Patient, Arzt, assistierende Person). Die anwesenden Personen (inkl. Patient) müssen einen Mundschutz tragen. Die Gespräche sollen auf die notwendigen Anweisungen für die Infiltration reduziert werden. Der Patient ist für die intraartikuläre Infiltration in Rückenlage. Intraartikuläre Infiltrationen werden an unserer Klinik unter Bildwandler-Kontrolle durchgeführt. Bei der subacromialen Infiltration, die an unserer Klinik ohne Bildwandler-Kontrolle durchgeführt wird, ist der Patient in sitzender Position. Es wird die posterolaterale Ecke des Acromions mit einem Stift markiert. Die Infiltrationsstelle befindet sich typischerweise $1 \mathrm{~cm}$ inferior und $1 \mathrm{~cm}$ medial dieser Ecke. Diese Stelle wird ebenfalls mit einem wasserfesten Stift markiert. Die desinfizierende Person soll vorher die Hände chirurgisch desinfizieren und anschliessend sterile Handschuhe tragen. Die assistierende Person reicht das notwendige Instrumentarium steril zu. Die Desinfektion erfolgt mit jodhaltigem Desinfektionsmittel mit $70 \%$ Alkoholanteil. Dabei wird dreimalig die komplette Region in einem Umkreis von $20 \mathrm{~cm}$ rund um die Infiltrationsstelle desinfiziert. Während des Trocknens des Desinfektionsmittels werden am «Back Table» die zu applizierenden Depots über eine eigens verwendete Kanüle aus den Ampullen steril in die Spritzen aufgezogen (Abb. 1). Nach Trockenen des Desinfektionsfelds wird ein steriles Tuch zur Abdeckung der benachbarten unsterilen Areale verwendet. Die Injektion erfolgt über eine eigene Infiltrationsna- 
del, die unmittelbar kurz vor der Infiltration steril ausgepackt wird (Abb. 2). Die Injektion soll kurz nach dem Trocknen des Desinfektionsmittels erfolgen. Bei der subacromialen Infiltration zieht die assistierende Person den Humerus nach distal. Dies gelingt am besten, wenn der Assistent den Ellenbogen des Patienten fasst und nach inferior zieht. Die Haut soll mit der Nadel schnell penetriert werden. Die Injektion erfolgt langsam. Die Patienten werden für 15 Minuten nach der Infiltration beobachtet. Bei der Infiltration von kurzwirksamem Lokalanästhetikum wird der Effekt der Infiltration erfragt. Dabei wird das Schmerzniveau auf einer Skala von 0-10 (0 = kein Schmerz, 10 = stärkster vorstellbarer Schmerz) vor der Infiltration und 15 Minuten nach der Infiltration erfasst und schriftlich festgehalten. Die Patienten sollten darüber informiert werden, dass der Lokalanästhetikum-Effekt nach etwa zwei Stunden (abhängig von dem applizierten Lokalanästhetikum) nachlässt und der Corticosteroid-Effekt erst nach zwei bis vier Tagen eintritt. Idealerweise wird der Patient über dieses Vorgehen und die entsprechenden Vorkehrungen im Vorfeld informiert.

Durch die Verbesserung der Schmerzsymptomatik wird die Schulterfunktion typischerweise auf ein akzeptables Mass verbessert [16]. Die Physiotherapie dient insbesondere für den Erhalt von Beweglichkeit und Kraft. Zudem sollen durch die Physiotherapie Begleitpathologien von benachbarten Gelenken, insbesondere dem scapulothorakalen Gelenk, verhindert werden, indem die physiologischen Bewegungsabläufe der Nachbargelenke kontrolliert und aufrechterhalten werden.

Bei der konservativen Behandlung ist es wichtig, dass Patienten über die natürliche Ruptur-Progression unter konservativer Therapie aufgeklärt werden. Es ist bekannt, dass insbesondere die schmerzhaften transmuralen Rupturen eine Progredienz der Rupturlänge und -breite von durchschnittlich 4 bzw. $2 \mathrm{~mm}$ pro Jahr aufweisen können [40]. Dementsprechend sollte bei Schmerztherapie trotz konservativer Therapiemassnahmen eine Arthro-MRI-Untersuchung innerhalb von sechs Monaten wiederholt werden, um eine Ruptur-Progression erkennen und entsprechend chir-

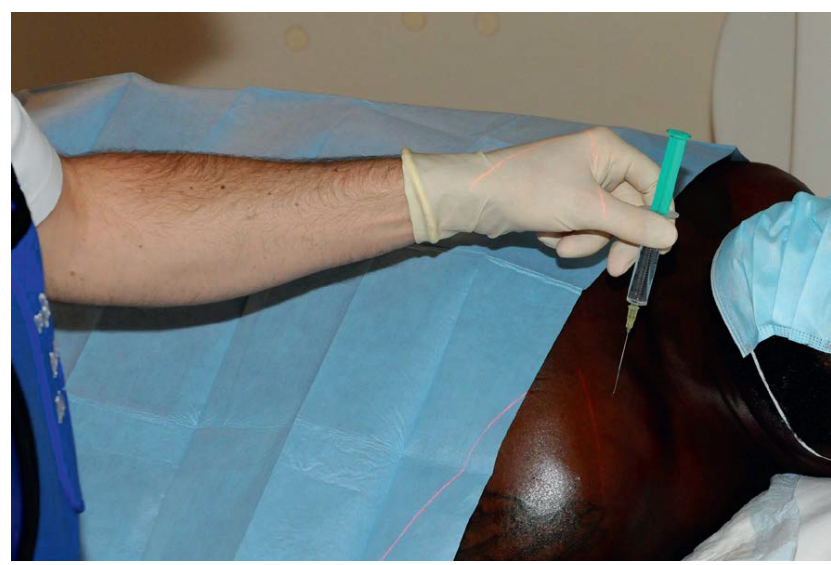

Abbildung 2. Bild des desinfizierten Infiltrationsgebietes. Patient mit Mundschutz. Sterile Handschuhe. Abdecken der unsterilen benachbarten Region. urgisch behandeln zu können. Die wesentlichsten Risikofaktoren für eine Ruptur-Progression sind: Nikotinabusus, Vorhandensein einer transmuralen Ruptur und die Rupturgrösse ( $>1 \mathrm{~cm}$ Länge und $>2 \mathrm{~cm}$ Breite) [40]. Für den klinischen Alltag in einer hausärztlichen Praxis kann folgende Regel für die konservative Behandlung von RM-Rupturen angewandt werden: So lange Patienten mit einer RM-Ruptur lediglich unter Schmerzen leiden und kein Kraftdefizit für Über-Kopf- oder Rotationsbewegungen im Raum aufweisen, kann die konservative Therapie der RM-Ruptur fortgesetzt werden. Tritt eine Schwäche bei den genannten Bewegungen auf, sollte der Patient an ein Schulterzentrum überwiesen werden. Dementsprechend ist es essenziell, Patienten bezüglich Schwäche bei Überkopfbewegungen $\mathrm{zu}$ befragen.

Es gibt keine klare Evidenz, wie häufig eine intraartikuläre oder subacromiale Infiltration erfolgen darf. An unserer Klinik wird im Rahmen des konservativen Therapieregimes maximal zweimal pro Jahr eine therapeutische intraartikuläre Infiltration durchgeführt. Das weitere therapeutische Procedere hängt dabei sehr von dem Ansprechen auf eine Infiltration ab. Kann zum Beispiel bei einem betagten Patienten durch eine Infiltration die Symptomatik für sechs Monate oder länger auf ein für den Patienten zufriedenstellendes Niveau gelindert werden, kann eine Infiltrationstherapie langfristig weiter durchgeführt werden. Ist hingegen bei einem aktiven Patienten die Infiltrationswirkung von zwei durchgeführten Infiltrationen kurz ( $<6$ Wochen) und der Leidensdruck entsprechend hoch, werden weitere therapeutische/chirurgische Möglichkeiten in Erwägung gezogen.

\section{Chirurgische Therapie}

Besteht der Leidensdruck trotz konservativer Therapie, muss mit Hilfe des MRIs geklärt werden, ob die Reparabilität der Ruptur noch gegeben ist. Bei jungen und aktiven Patienten mit hohem Funktionsanspruch, reparabler Ruptur und entsprechend hohem Leidensdruck ist die primär chirurgische Rekonstruktion in der Regel zu favorisieren [10, 41]. Diese kann arthroskopisch oder offen erfolgen. Auch wenn mehrere Studien keine Überlegenheit der arthroskopischen gegenüber der offenen RM-Rekonstruktion nachweisen konnten [42, 43], werden RM-Rekonstruktionen heutzutage mehrheitlich, aufgrund der geringeren Morbidität, besseren Kosmetik und besseren Gelenksvisualisierung, arthroskopisch durchgeführt [44] - siehe Fallbeispiel 1. Bei offenen wie geschlossenen Operationsverfahren wird die abgerissene Sehne mit Hilfe von verschiedenen Fadenstichtechniken [45-48] gefasst und dann mittels transossärem Fadendurchzug oder über ein intrasossär eingebrachtes Ankersystem an den Humerus refixiert. Das Heranführen der Sehne an ihren ursprünglichen ossären Ansatzpunkt garantiert noch keine Heilung der Sehne. Die postoperative Nachbehandlung mit Schonung des Schultergelenks ist neben der Operationstechnik und den biologischen Voraussetzungen ausschlagge- 
bend für eine erfolgreiche Sehnen-zu-Knochen-Heilung. Da die exakte Dauer bis zur Sehnen-Knochen-Heilung nicht vollends bekannt und zudem individuell unterschiedlich ist, existiert eine Vielzahl von Nachbehandlungsprotokollen nach RM-Rekonstruktionen. Die Rehabilitation, wie sie auch an unserer Klinik Anwendung findet, kann in vier Phasen eingeteilt werden [49]. In der ersten Phase (Wochen 0-6) wird die Rekonstruktion geschützt, indem der operierte Arm geschont (Schlinge oder Abduktionsschiene) und lediglich passiv (durch Physiotherapeuten) bewegt wird. Phase 2 (Wochen 6-12) besteht aus zunehmend aktiven Bewegungsübungen mit Körper-/Armeigengewicht (aber ohne zusätzliches Gewicht). In Phase 3 (Wochen 13-18) kann mit sanfter Kräftigung und in Phase 4 (ab Woche 19) mit forcierterem Training/ Muskelaufbau begonnen werden. Bei gutem Verlauf ist in der Regel die Rehabilitationszeit von vier bis sechs Monaten ausreichend. Das Endresultat ist nach einem Jahr erreicht. Patienten, die körperlich schwer arbeiten müssen, benötigen gelegentlich längere Rehabilitationszeiten. Es ist wichtig, die Arbeitsunfähigkeit mit den Patienten im Vorfeld zu diskutieren, sodass bei Bedarf der Arbeitgeber vom Patienten frühzeitig informiert werden kann, um eine Konfliktsituation zu vermeiden. Im Rehabilitationsverlauf ist es nützlich, wenn der Spezialist die Arbeitsunfähigkeit festlegt und somit den Hausarzt unterstützt. Ist aufgrund negativer Erfahrungen aus vorangegangen Therapien, hohem sozialem Druck am Arbeitsplatz oder einer schwierigen psychischen Konstitution eine niedrige Compliance vorhersehbar, muss eine operative Therapie mit der damit verbundenen, sehr aufwändigen Nachbehandlung in Frage gestellt werden.

Ist die RM-Ruptur irreparabel und der Funktionsanspruch des Patienten hoch, ist je nach Typ der Läsion die Indikation entweder für einen Muskel-Sehnen-Transfer, die Implantation einer inversen Schultertotalprothese [50] oder die Kombination aus beidem zu prüfen [51]. Folgende Muskel-Sehnen-Einheiten werden zur Kompensation von Elevation und Aussenrotationsschwäche bei vorliegender posterosuperioren RM-Ruptur (Supraspinatus und Infraspinatus, ggf. mit Teres minor) verwendet: Latissimus-dorsi-Transfer [52, 53] - siehe Fallbeispiel 2, Teres major [54] oder inferiorer Trapezius-Transfer [55]. Zur Behandlung der Innenrotationsschwäche bei irreparabler anteriorer RM-Ruptur (Subscapularis) stehen der etablierte Pectoralis-major- und neuerdings auch der anteriore Latissimus-dorsi-Transfer zur Verfügung. Von den beschriebenen Sehnentransfers existieren nur für den Latissimus dorsi-Transfer [56] und den Pectoralis-majorTransfer [57] Langzeitergebnisse, die jedoch, in Anbetracht der üblicherweise schwierigen Ausgangslage, als durchaus zufriedenstellend einzustufen sind.

Ist die irreparable RM-Ruptur von einer Omarthrose begleitet oder besteht eine irreparable Massenruptur mit Beteiligung sowohl der anterioren als auch posterioren RM, ist eine gelenkserhaltende Sehnenrekonstruktion mit schlechteren Behandlungsergebnissen verbunden. Diese Patienten qualifizieren in diesen Fällen für die Implantation einer in-

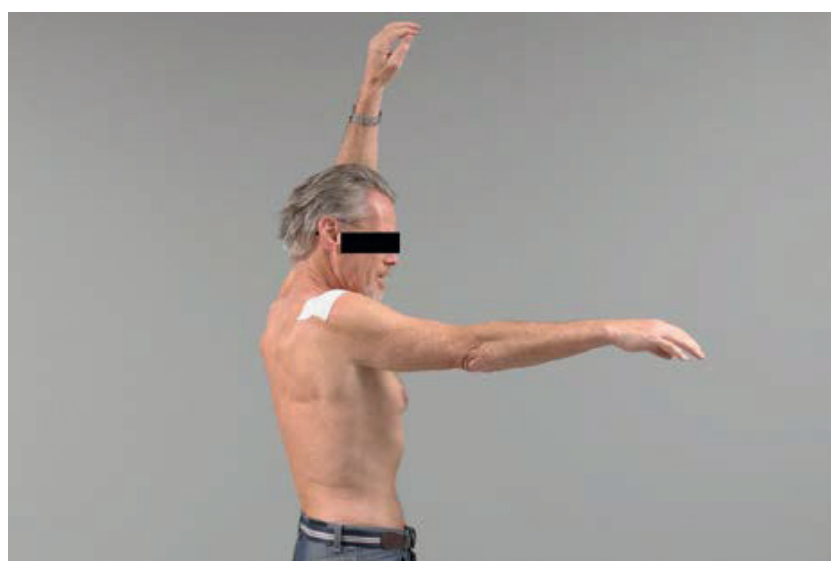

Abbildung 3. Fallbeispiel 1, Funktionsaufall des rechten Arms.



Abbildung 4. Arthro-MRI: Ruptur der Supraspinatussehne (Pfeil zeigt auf den retrahierten Supraspinatussehnenstumpf).

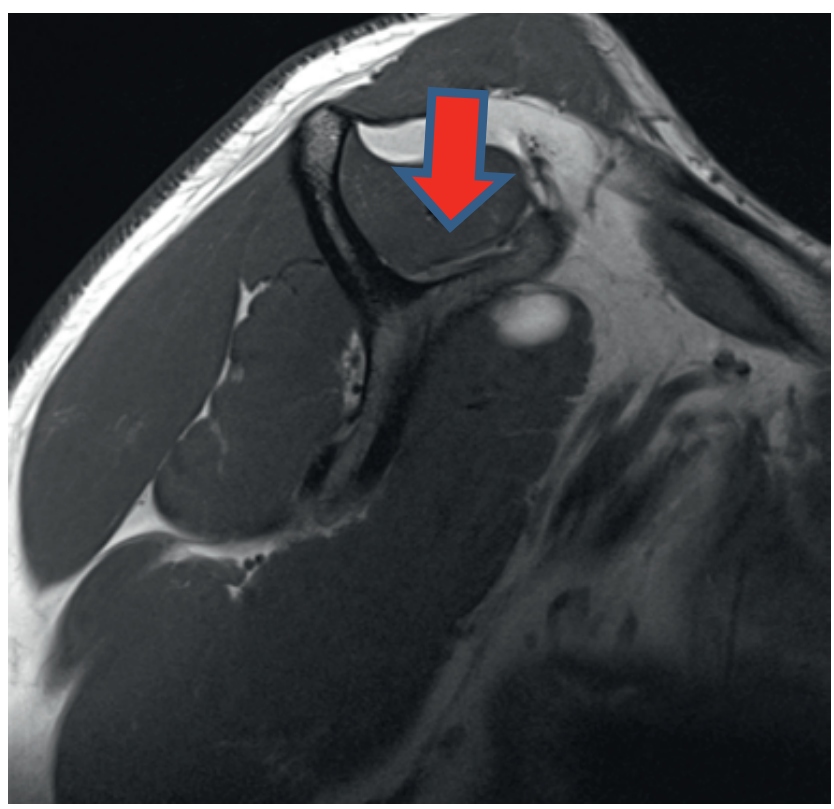

Abbildung 5. Parasagittaler Schnitt durch die Muskelbäuche der RM-Muskeln. Pfeil: Suraspinatusmuskel. 


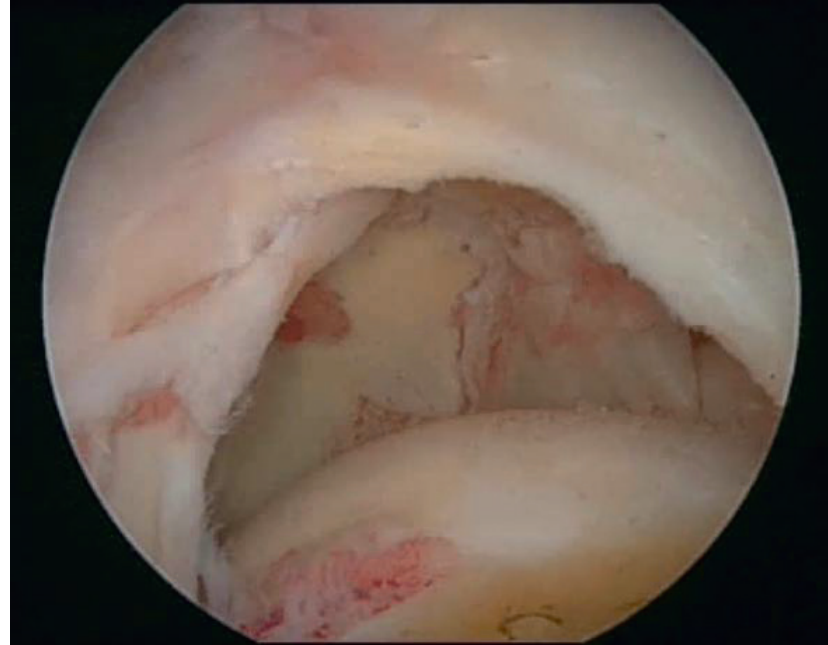

Abbildung 6. Supraspinatussehne.

versen Schulterprothese [58]. Die Schulterfunktion und die Lebensqualität können bei diesen Patienten wesentlich und auch langfristig verbessert werden [50]. Bei Patienten mit schlechten biologischen Grundvoraussetzungen ist die Chance für erfolgreiche Sehnenheilung geringer. Dementsprechend sind Patienten mit z.B. rheumatoider Arthritis häufiger von Re-Rupturen nach Sehnenrekonstruktionen betroffen und profitieren insbesondere bei höherem Patientenalter von einer Behandlung der RM-Ruptur mittels inverser Schulterprothese [59, 60]. Dasselbe gilt für Patienten mit mehrfach fehlgeschlagenen RM-Rekonstruktionen oder Patienten nach fehlgeschlagenen Sehnentransferoperationen. Besteht bei einem Patienten mit irreparabler RMRuptur neben der Elevationsschwäche auch ein Ausfall der Aussenrotationsfähigkeit bzw. der Haltefähigkeit im Raum, profitieren die Patienten von der Implantation einer inversen Schulterprothese in Kombination mit einem Latissimus-dorsi-Transfer [51] - siehe Fallbeispiel 4 oder in Kombination Latissimus-dorsi- und Teres-major- Transfer [61]. Irreparable Sehnenrupturen bei Patienten mit niedrigen Funktionsansprüchen oder schlechtem Allgemeinzustand werden wenn möglich konservativ behandelt und zeigen bei guter Schmerzkontrolle akzeptable Ergebnisse sowohl bei grossen [16] wie bei kleinen Rupturen [15].

\section{Fallbeispiel 1}

Der 67-jährige pensionierte Patient stellt sich bei seinem Hausarzt mit seit fünf Jahren bestehenden Schulterschmerzen vor. Seit einem Bagatelltrauma (Kontusion im Zweikampf), das sich der Patient beim wöchentlichen Fussballspielen vor zwölf Wochen zugezogen hatte, kann er den rechten Arm nicht mehr über die Horizontale heben. Nach erfolgloser Physiotherapieserie und zunehmender Schwäche, kommt der Patient in unsere Sprechstunde und zeigt in der klinischen Untersuchung eine deutliche Schwäche und Funktionsausfall des rechten dominanten Arms (Abb. 3).
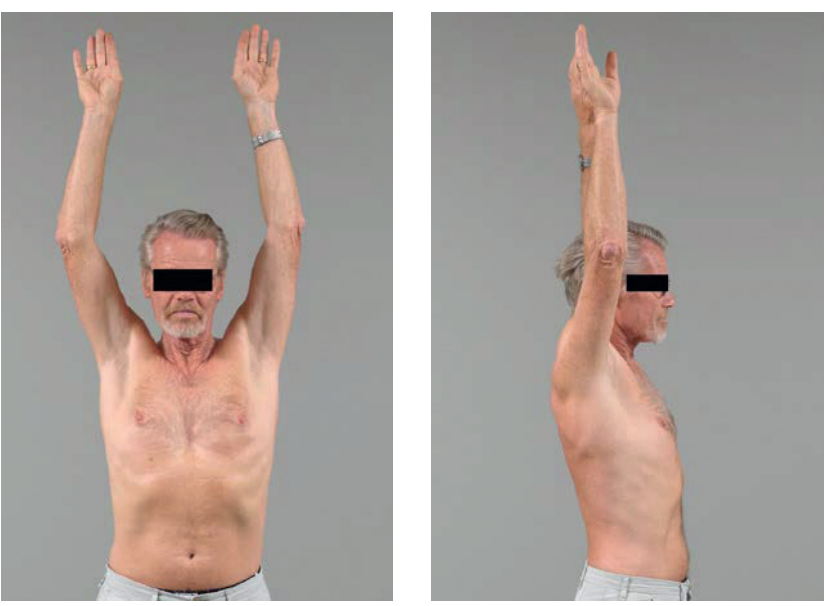

Abbildungen 7, 8. Ein Jahr postoperativ.

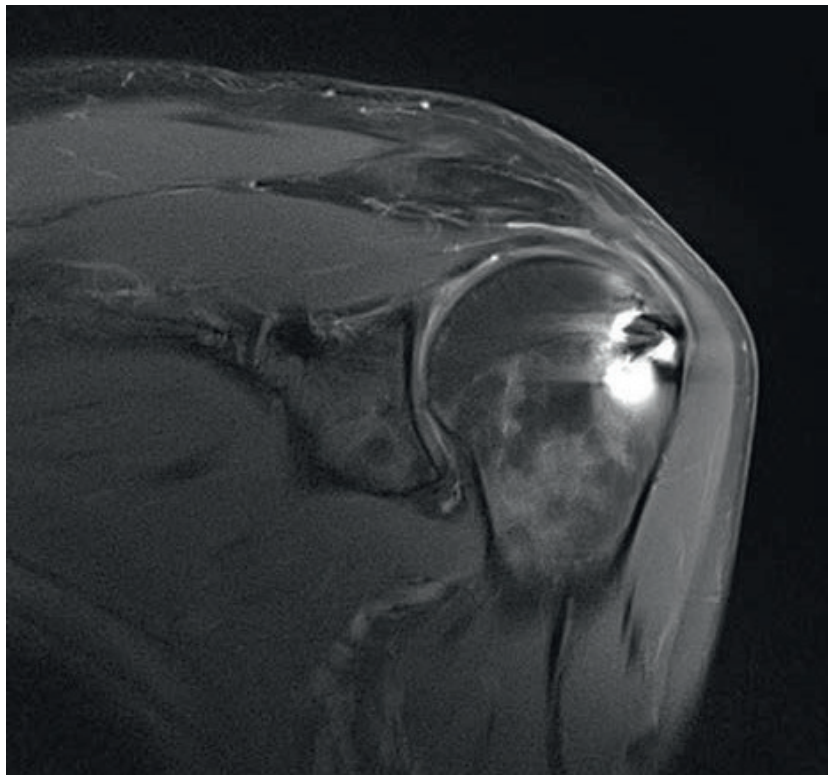

Abbildung 9. Supraspinatussehne, MRI.

Der Supraspinatusmuskel (Abb. 5, roter Pfeil) zeigt eine gute Muskelqualität, ohne Degeneration. Dementsprechend ist die Ruptur bei guter Muskelqualität reparabel. Abbildung 6 zeigt arthroskopisch den U-förmigen Defekt in der Supraspinatussehne. Bei noch akzeptabler Muskelqualität erfolgte eine arthroskopische Rekonstruktion der Rotatorenmanschette.

Ein Jahr postoperativ ist der Patient beschwerdefrei und kann seinen sportlichen Aktivitäten uneingeschränkt nachgehen. Abbildungen 7 und 8 zeigen eine uneingeschränkte Schulterbeweglichkeit und eine geheilte Supraspinatussehne in der MRI-Untersuchung (Abb. 9).

\section{Fallbeispiel 2}

57-jähriger Patient mitirreparabler Rotatorenmanschettenruptur der rechten dominanten Schulter. Der Patient ist vor allem durch die Schwäche, bzw. die Unfähigkeit für Überkopfbewegungen, gestört (Abb. 10). 
Aufgrund der Irreparabilität (Verfettung der SupraspinatusMuskel Goutallier Grad III und Infraspinatusmuskulatur Goutallier IV - rote Pfeile, Abb. 11) wird ein Latissimus-dor-

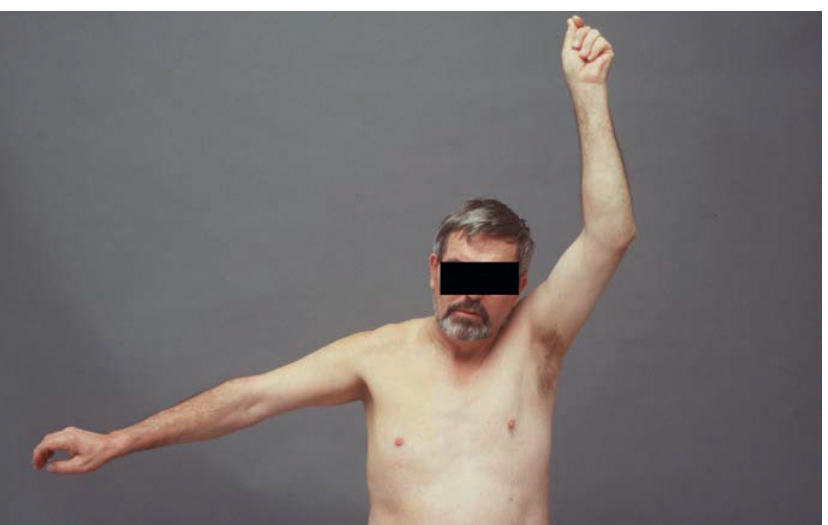

Abbildung 10. 57-jähriger Patient mit irreparabler RM-Ruptur und Abduktionsefizit rechts.

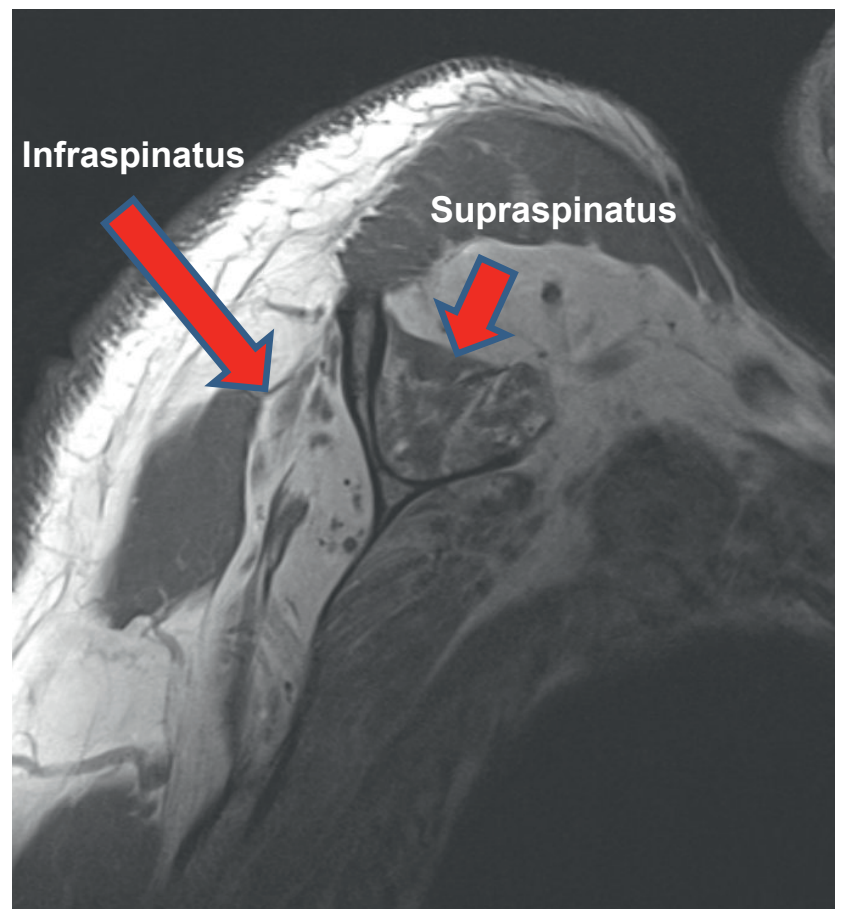

Abbildung 11. Supraspinatus-Muskel.
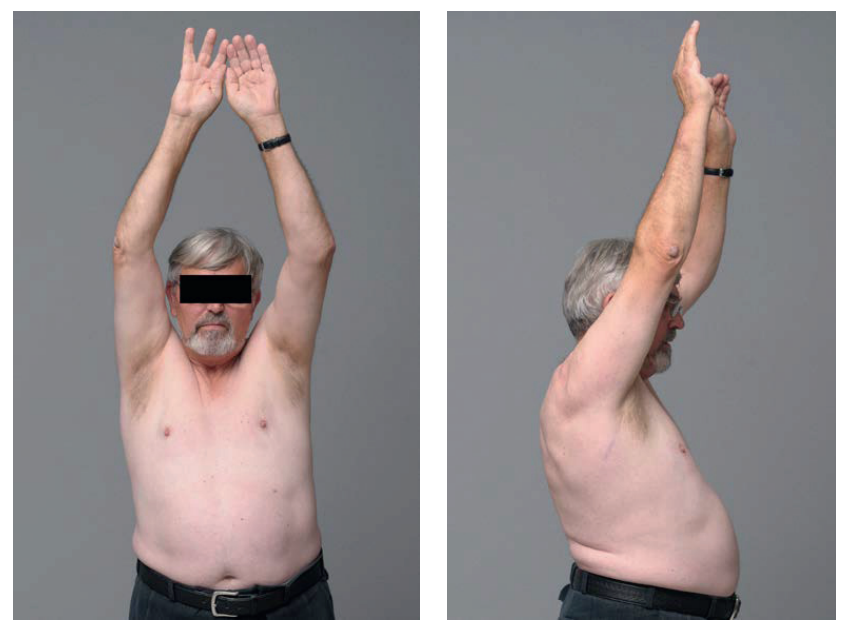

Abbildungen 12, 13. Patient 2, zehn Jahre postoperativ.
si-Sehnentransfer durchgeführt, um die Überkopfschwäche zu behandeln.

Zehn Jahre nach der Operation ist der Patient weiterhin beschwerdefrei und kann die Schulter uneingeschränkt bewegen und beanspruchen (Abb. 12, 13).

\section{Fallbeispiel 3}

76-jährige Patientin mit einer irreparablen RM-Ruptur mit fehlender Elevation und komplettem Aussenrotationsausfall (Abb. 14-16).

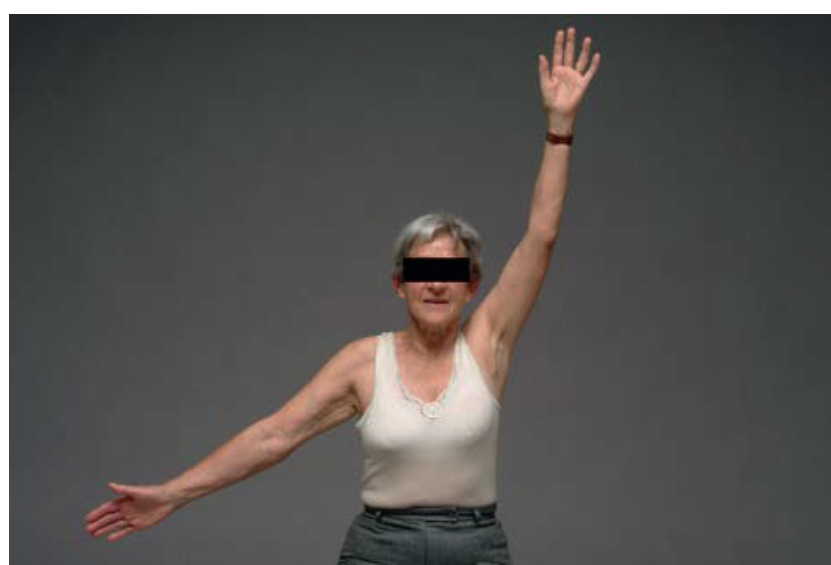

Abbildung 14. 76-jährige Patientin mit fehlender Abduktionsfähigkeit rechts bei irreparabler RM-Ruptur.

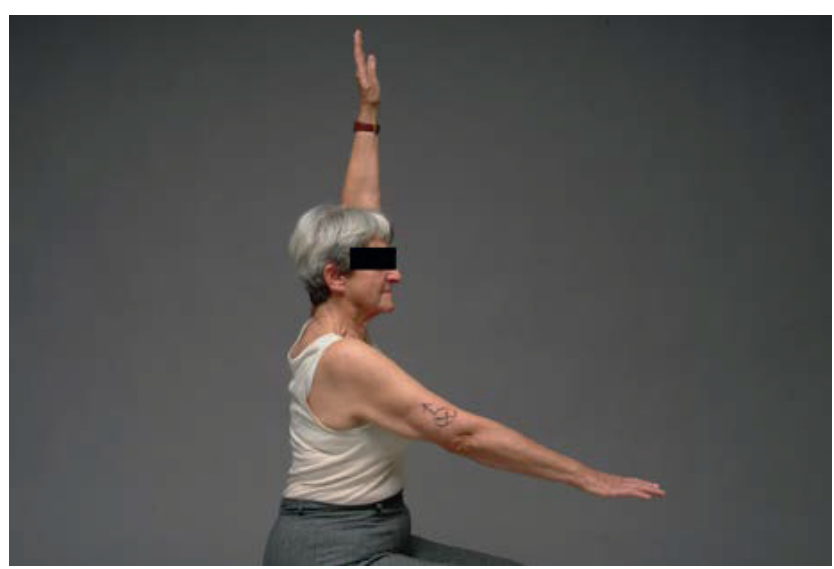

Abbildung 15. Elevationsverlust rechter Arm.

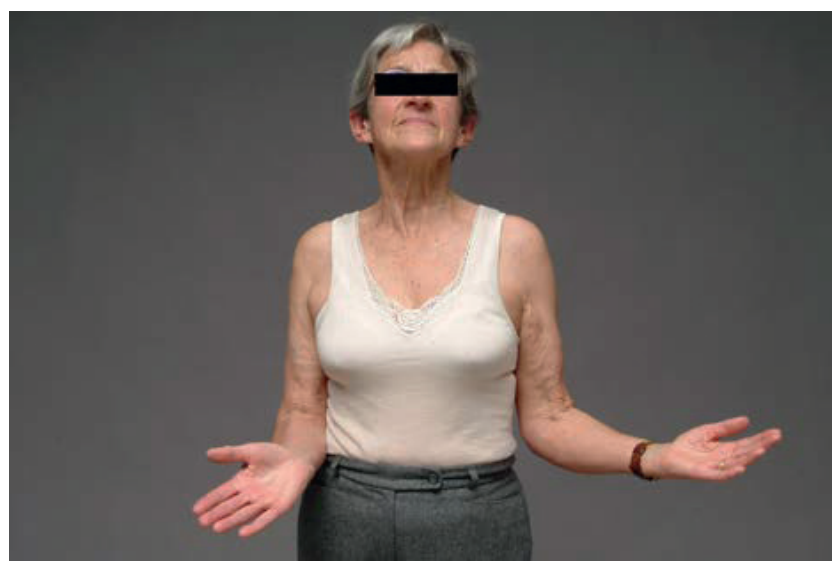

Abbildung 16. Aussenrotationsdefizit der rechten Schulter. 


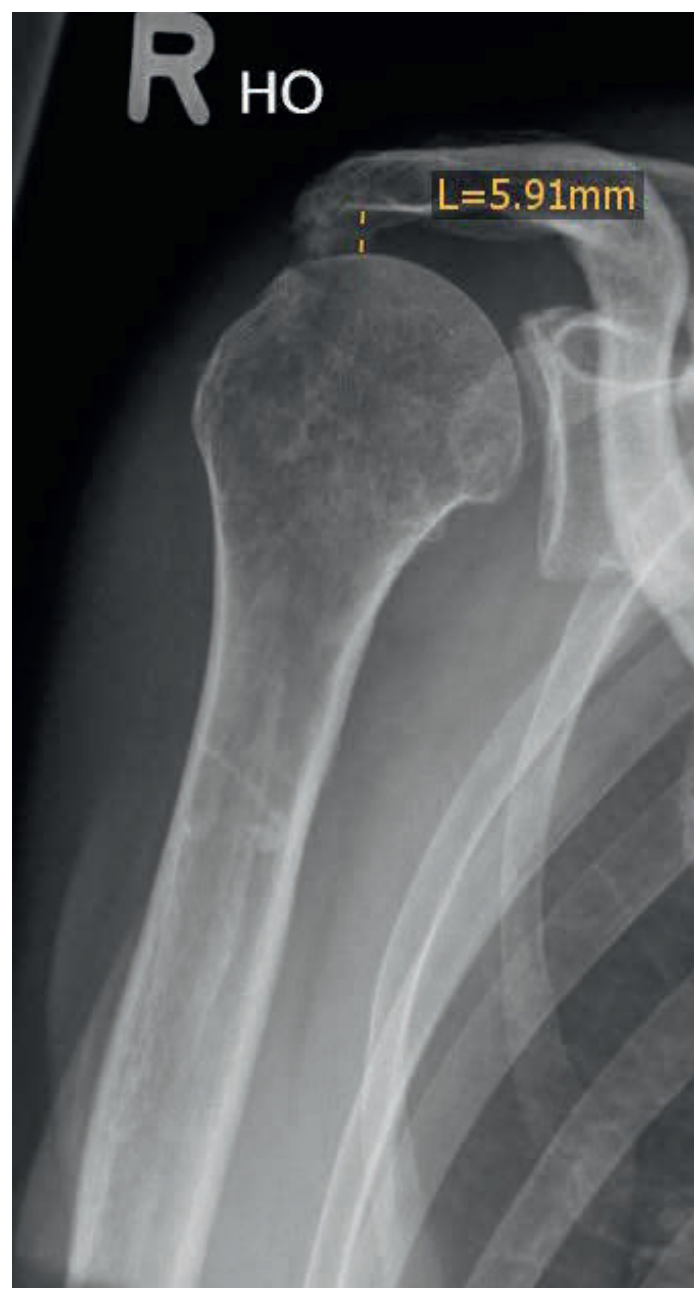

Abbildung 17. Schulter vor Operation, Fallbericht Nr. 3.
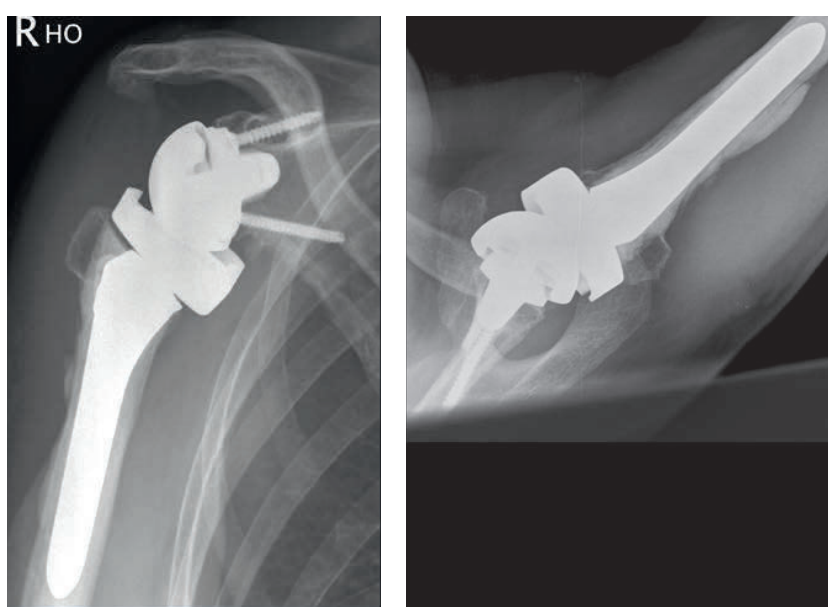

Abbildungen 18, 19. Inverse Schulterprothese.

Aufgrund des kombinierten Ausfalls von Aussenrotation- und Elevationsfunktion der rechten Schulter wurde die Patientin mittels einer inversen Schulterprothese in Kombination mit einem Latissimus-dorsi-Transfer behandelt. Röntgenbild der Schulter vor der Operation mit vermindertem ACHD-Abstand von $<7 \mathrm{~mm}$ (Abb. 17) und postoperativen Bilder mit Implantation der inversen Schulterprothese (Abb. 18, 19).
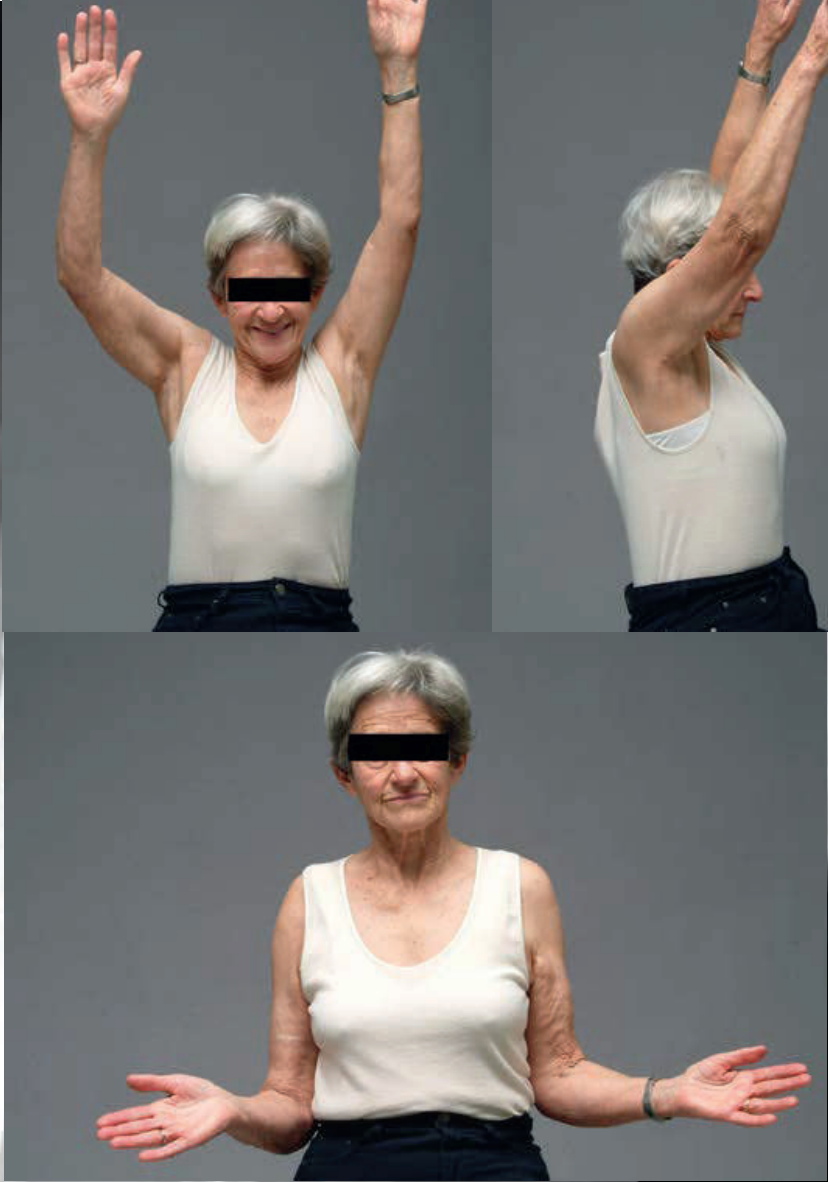

Abbildungen 20, 21, 22. Fallbericht Nr. 3, zwölf Jahre postoperativ. Die Patientin ist schmerzfrei und zeigt eine gute Schulterfunktion.

\section{Bibliografie}

1. Milgrom C, Schaffler M, Gilbert S, van Holsbeeck M. Rotator-cuff changes in asymptomatic adults. The effect of age, hand dominance and gender. J Bone Joint Surg Br 1995; 77: 296-298.

2. Yamamoto A, Takagishi K, Osawa T, et al.: Prevalence and risk factors of a rotator cuff tear in the general population. J Shoulder Elbow Surg 2010; 19: 116-120.

3. Cowan JB, Bedi A, Carpenter JE, Robbins CB, Gagnier JJ, Miller BS: Evaluation of American Academy of Orthopaedic Surgeons Appropriate Use Criteria for the management of full-thickness rotator cuff tears. J Shoulder Elbow Surg 2016; 25: 1100-1106.

4. Murray J, Gross L: Optimizing the management of full-thickness rotator cuff tears. J Am Acad Orthop Surg 2013; 21: 767-771.

5. Surgeons AAoO: Appropriate use criteria for optimizing the management of full-thickness rotator cuff tears. http://www. aaos.org/research/Appropriate_Use/rotatorcuffaucfull. pdf:2013; letzter Zugriff 15.01.2019.

6. Acevedo DC, Paxton ES, Williams GR, Abboud JA: A survey of expert opinion regarding rotator cuff repair. J Bone Joint Surg Am 2014; 96: e123.

7. Elhassan BT, Cox RM, Shukla DR, Lee J, Murthi AM, Tashjian RZ, Abboud JA: Management of failed rotator cuff repair in young patients. J Am Acad Orthop Surg 2017; 25: e261-e271.

8. Jost B, Pfirrmann CW, Gerber C, Switzerland Z: Clinical outcome after structural failure of rotator cuff repairs. J Bone Joint Surg Am 2000; 82: 304-314.

9. Galatz LM, Ball CM, Teefey SA, Middleton WD, Yamaguchi K: The outcome and repair integrity of completely arthroscopically repaired large and massive rotator cuff tears. J Bone Joint Surg Am 2004; 86A: 219-224. 


\section{Key messages}

- Anhand der Anamnese können durch Differenzierung der Hauptsymptome Schmerz oder Schwäche meist die Weichen für die Therapie gestellt werden. Schulterschmerzen alleine können mittels Neer-Test weiter abgeklärt werden. Sind die Schmerzen nach subacromialer Infiltration von Lokalanästhetikum nicht mehr vorhanden und eine Schwäche kann im Zustand der Schmerzfreiheit ausgeschlossen werden, ist eine konservative Therapie möglich. Zeigt sich hingegen eine Schwäche, kann diese ein Hinweis für eine RM-Ruptur darstellen. Bei jungen Patienten und Patienten mit hohem Anspruchsprofil sollte beim Nachweis einer Ruptur diese eher operativ versorgt werden.

- Die Erhebung des Aktivitätsprofils des Patienten in der Arbeit und in der Freizeit ist für die Entscheidung, ob eine konservative oder operative Behandlung durchgeführt werden soll, ausschlaggebend.

- Pseudoparalyse, Aussenrotation- und Innenrotationslag-Zeichen, positiver Jobe-, Whipple-, Lift-off-, Belly-PressTest und Hornblower-Zeichen sind klinische Untersuchungsbefunde, die auf eine mechanisch relevante Rotatorenmanschettenläsion hinweisen, die bei aktiven oder jungen Patienten mit entsprechender störender Funktionseinschränkung operativ behandelt werden sollten.

- Eine im anteroposterioren Röntgenbild der Schulter acromiohumerale Distanz von <7 mm ist ein Hinweis für eine chronische posterosuperiore Rotatorenmanschettenruptur, die nicht mehr rekonstruierbar ist.

- RM-Rupturen mit magnetresonanztomografisch höhergradiger Verfettung der Rotatorenmanschettenmuskulatur sind mit einer hohen Rerupturrate im Falle von RMR verbunden und werden daher ebenfalls als irreparable Rotatorenmanschettenrupturen beurteilt.

\section{Lernfragen}

1. Welche der anamnestischen Angaben ist am charakteristischsten für eine Rotatorenmanschettenruptur? (Einfachauswahl)

a) Schwäche bei Schulterbewegungen

b) Schulterschmerzen bei Alltagsaktivitäten

c) Schulterschmerzen bei sportlichen Aktivitäten

d) Bewegungseinschränkung für Innenrotation

e) Bewegungseinschränkung für Aussenrotation

2. Eine Rotatorenmanschettenruptur gilt als operativ irreparabel, wenn ...(Einfachauswahl)

a) ...eine transmurale Ruptur vorliegt.

b) ...eine Partialruptur vorliegt.

c) ...eine magnetresonanztomografische Verfettung der Rotatorenmanschettenmuskulatur Goutallier I oder || vorliegt.

d) ...eine magnetresonanztomografische Verfettung der Rotatorenmanschettenmuskulatur Goutallier III oder IV vorliegt.

e) ...eine höhergradige Retraktion des Sehnenstumpfes ohne muskuläre Verfettung vorliegt.

3. Welche der folgenden Therapieoptionen eignet sich für die Behandlung einer irreparablen Rotatorenmanschettenruptur am wenigsten? (Einfachauswahl)
a) Latissimus-dorsi-Sehnentransfer
b) Pectoralis-major-Sehnentransfer
c) Implantation einer inversen Schulterprothese
d) Inferiorer Trapezius-Sehnentransfer
e) Arthroskopische Rotatorenmanschettenrekonstruktion

10. Lafosse L, Brozska R, Toussaint B, Gobezie R: The outcome and structural integrity of arthroscopic rotator cuff repair with use of the double-row suture anchor technique. J Bone Joint Surg Am 2007; 89: 1533-1541.

11. Gilbart MK, Gerber C: Comparison of the subjective shoulder value and the Constant score. J Shoulder Elbow Surg 2007; 16:717-721.

12. Fuchs S, Chylarecki C, Langenbrinck A. Incidence and symptoms of clinically manifest rotator cuff lesions. Int J Sports Med 1999; 20: 201-205.

13. Gerber C: Massive rotator cuff tears. In: Iannotti JP (ed). Disorders of the shoulder. Diagnosis and managment. 1st ed. Philadelphia: 1999.

14. Lahteenmaki HE, Virolainen P, Hiltunen A, Heikkila J, Nelimarkka OI: Results of early operative treatment of rotator cuff tears with acute symptoms. J Shoulder Elbow Surg 2006; 15:148-153.
15. Fucentese SF, von Roll AL, Pfirrmann CW, Gerber C, Jost B: Evolution of nonoperatively treated symptomatic isolated fullthickness supraspinatus tears. J Bone Joint Surg Am 2012; 94: 801-808.

16. Zingg PO, Jost B, Sukthankar A, Buhler M, Pfirrmann CW, Gerber $\mathrm{C}$ : Clinical and structural outcomes of nonoperative management of massive rotator cuff tears. J Bone Joint Surg Am 2007; 89: 1928-1934.

17. Ellman H, Hanker G, Bayer M: Repair of the rotator cuff. Endresult study of factors influencing reconstruction.J Bone Joint Surg Am 1986; 68: 1136-1144.

18. Gerber C, Wirth SH, Farshad M: Treatment options for massive rotator cuff tears. J Shoulder Elbow Surg 2011; 20: S20-29.

19. Goutallier D. PD, Lavau L, Bernageau J: Influence de la dégénérescence graisseuse des muscles supraépineux et infraépineux 
sur le pronostic des réparations chirurgicales de la coiffe des rotateurs. Revue de chirurgie orthopédique et réparatrice de l'appareil moteur 1999; 85: 668-676.

20. Gerber C, Krushell RJ: Isolated rupture of the tendon of the subscapularis muscle. Clinical features in 16 cases. J Bone Joint Surg Br 1991; 73: 389-394.

21. Werner CM, Steinmann PA, Gilbart M, Gerber C: Treatment of painful pseudoparesis due to irreparable rotator cuff dysfunction with the Delta III reverse-ball-and-socket total shoulder prosthesis. J Bone Joint Surg Am 2005; 87: 1476-1486.

22. Hertel R, Ballmer FT, Lombert SM, Gerber C: Lag signs in the diagnosis of rotator cuff rupture. J Shoulder Elbow Surg 1996; 5:307-313.

23. Walch G, Boulahia A, Calderone S, Robinson AH: The 'dropping' and 'hornblower's' signs in evaluation of rotator-cuff tears. J Bone Joint Surg Br 1998; 80: 624-628.

24. Jain NB, Wilcox RB, 3rd, Katz JN, Higgins LD: Clinical examination of the rotator cuff. PM R 2013; 5: 45-56.

25. Jobe FW, Moynes DR: Delineation of diagnostic criteria and a rehabilitation program for rotator cuff injuries. Am J Sports Med 1982; 10:336-339.

26. Savoie FH, 3rd, Field LD, Atchinson S: Anterior superior instability with rotator cuff tearing: SLAC lesion. The Orthopedic clinics of North America 2001; 32: 457-461.

27. Sgroi M, Loitsch T, Reichel H, Kappe T: Diagnostic Value of Clinical Tests for Supraspinatus Tendon Tears. Arthroscopy: the journal of arthroscopic \& related surgery : official publication of the Arthroscopy Association of North America and the International Arthroscopy Association: 2018.

28. Barth JR, Burkhart SS, De Beer JF: The bear-hug test: a new and sensitive test for diagnosing a subscapularis tear. Arthroscopy 2006; 22: 1076-1084.

29. Neer CS: Shoulder reconstruction. Philadelphia; Saunders: 1990

30. Neer CS, $2^{\text {nd }}$ : Impingement lesions. Clin Orthop Relat Res 1983: 70-77.

31. Gruber G, Bernhardt GA, Clar H, Zacherl M, Glehr M, Wurnig C Measurement of the acromiohumeral interval on standardized anteroposterior radiographs: a prospective study of observer variability. J Shoulder Elbow Surg 2010; 19: 10-13.

32. Gerber C, Fuchs B, Hodler J: The results of repair of massive tears of the rotator cuff. J Bone Joint Surg Am 2000; 82: 505-515.

33. Lenza M, Buchbinder R, Takwoingi Y, Johnston RV, Hanchard NC, Faloppa F: Magnetic resonance imaging, magnetic resonance arthrography and ultrasonography for assessing rotator cuff tears in people with shoulder pain for whom surgery is being considered. Cochrane Database Syst Rev 2013: CD009020.

34. Ellman H. Diagnosis and treatment of incomplete rotator cuff tears. Clin Orthop Relat Res 1990: 64-74.

35. DeOrio JK, Cofield RH: Results of a second attempt at surgical repair of a failed initial rotator-cuff repair. J Bone Joint Surg Am 1984; 66: 563-567.

36. Patte D: Classification of rotator cuff lesions. Clin Orthop Relat Res 1990: 81-86.

37. Fuchs B, Weishaupt D, Zanetti M, Hodler J, Gerber C: Fatty degeneration of the muscles of the rotator cuff: assessment by computed tomography versus magnetic resonance imaging. $J$ Shoulder Elbow Surg 1999; 8: 599-605.

38. Goutallier D, Postel JM, Bernageau J, Lavau L, Voisin MC: Fatty muscle degeneration in cuff ruptures. Pre- and postoperative evaluation by CT scan. Clin Orthop Relat Res 1994: 78-83.

39. Goutallier D, Postel JM, Gleyze P, Leguilloux P, Van Driessche S: Influence of cuff muscle fatty degeneration on anatomic and functional outcomes after simple suture of full-thickness tears. J Shoulder Elbow Surg 2003; 12: 550-554.

40. Yamamoto N, Mineta M, Kawakami J, Sano H, Itoi E: Risk ractors for tear progression in symptomatic rotator cuff tears: A prospective study of 174 shoulders. Am J Sports Med 2017; 45: 2524-2531.

41. Collin P, KempfJF, Mole D, Meyer N, Agout C, Saffarini M, Godeneche A: Société Française de Chirurgie Orthopédique et Traumatologique (SoFCOT): Ten-year multicenter clinical and MRI eval- uation of isolated supraspinatus repairs. J Bone Joint Surg Am 2017; 99: 1355-1364

42. Bishop J, Klepps S, Lo IK, Bird J, Gladstone JN, Flatow EL: Cuff integrity after arthroscopic versus open rotator cuff repair: a prospective study. J Shoulder Elbow Surg 2006; 15: 290-299.

43. Morse K, Davis AD, Afra R, Kaye EK, Schepsis A, Voloshin I: Arthroscopic versus mini-open rotator cuff repair: a comprehensive review and meta-analysis. Am J Sports Med 2008; 36: 1824-1828.

44. Colvin AC, Egorova N, Harrison AK, Moskowitz A, Flatow EL: National trends in rotator cuff repair. J Bone Joint Surg Am 2012; 94: 227-233

45. Gerber C, Schneeberger AG, Beck M, Schlegel U: Mechanical strength of repairs of the rotator cuff. J Bone Joint Surg Br 1994; 76: 371-380.

46. Gerber C, Schneeberger AG, Perren SM, Nyffeler RW: Experimental rotator cuff repair. A preliminary study. J Bone Joint Surg Am 1999; 81: 1281-1290.

47. Schneeberger AG, Beck M, Gerber C: [In-vitro stability of rotator cuff repair techniques]. Orthopade 1995; 24: 268-274.

48. Schneeberger AG, von Roll A, Kalberer F, Jacob HA, Gerber C: Mechanical strength of arthroscopic rotator cuff repair techniques: an in vitro study. J Bone Joint Surg Am 2002; 84-A: 2152-2160.

49. Millett PJ, Wilcox RB, 3rd, O'Holleran JD, Warner JJ: Rehabilitation of the rotator cuff: an evaluation-based approach. J Am Acad Orthop Surg 2006; 14: 599-609.

50. Gerber C, Canonica S, Catanzaro S, Ernstbrunner L: Longitudinal observational study of reverse total shoulder arthroplasty for irreparable rotator cuff dysfunction: results after 15 years. J Shoulder Elbow Surg 2018; 27: 831-838.

51. Puskas GJ, Catanzaro S, Gerber C: Clinical outcome of reverse total shoulder arthroplasty combined with latissimus dorsi transfer for the treatment of chronic combined pseudoparesis of elevation and external rotation of the shoulder. J Shoulder Elbow Surg 2014; 23: 49-57.

52. Gerber C: Latissimus dorsi transfer for the treatment of irreparable tears of the rotator cuff. Clin Orthop Relat Res 1992: 152-160.

53. Gerber C, Vinh TS, Hertel R, Hess CW: Latissimus dorsi transfer for the treatment of massive tears of the rotator cuff. A preliminary report. Clin Orthop Relat Res 1988: 51-61.

54. Wirth MA, Rockwood CA, Jr: Operative treatment of irreparable rupture of the subscapularis. J Bone Joint Surg Am 1997; 79 $722-731$.

55. Elhassan BT, Alentorn-Geli E, Assenmacher AT, Wagner ER: Arthroscopic-assisted lower trapezius tendon transfer for massive irreparable posterior-superior rotator Cuff tears: surgical technique. Arthrosc Tech 2016; 5: e981-e988.

56. Gerber C, Rahm SA, Catanzaro S, Farshad M, Moor BK: Latissimus dorsi tendon transfer for treatment of irreparable posterosuperior rotator cuff tears: long-term results at a minimum follow-up of ten years. J Bone Joint Surg Am 2013; 95: 1920-1926.

57. Moroder P, Schulz E, Mitterer M, Plachel F, Resch H, Lederer S: Long-Term Outcome After Pectoralis Major Transfer for Irreparable Anterosuperior Rotator Cuff Tears. J Bone Joint Surg Am 2017; 99: 239-245

58. Grammont PM, Baulot E: Delta shoulder prosthesis for rotator cuff rupture. Orthopedics 1993; 16: 65-68.

59. Holcomb JO, Hebert DJ, Mighell MA, Dunning PE, Pupello DR, Pliner MD, Frankle MA: Reverse shoulder arthroplasty in patients with rheumatoid arthritis. J Shoulder Elbow Surg 2010 19: 1076-1084.

60. Rittmeister M, Kerschbaumer F: Grammont reverse total shoulder arthroplasty in patients with rheumatoid arthritis and nonreconstructible rotator cuff lesions. J Shoulder Elbow Surg 2001; 10: 17-22.

61. Shi LL, Cahill KE, Ek ET, Tompson JD, Higgins LD, Warner JJ: Latissimus Dorsi and Teres Major Transfer With Reverse Shoulder Arthroplasty Restores Active Motion and Reduces Pain for Posterosuperior Cuff Dysfunction. Clin Orthop Relat Res 2015 473: $3212-3217$ 
Manuskript eingereicht: 21.09.2018

Manuskript akzeptiert: 10.12.2018

Interessenskonflikt: Die Autoren erklären, dass keine Interessenskonflikte bestehen.

\section{Dr. med. Florian Grubhofer}

Oberarzt Orthopädie

Universitätsklinik Balgrist

Forchstrasse 340

8008 Zürich

florian.grubhofer@balgrist.ch

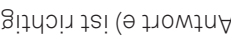

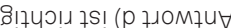

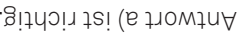

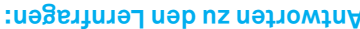

(1)

\section{Funktionsbeurteilung bei Kindern und Jugendlichen}

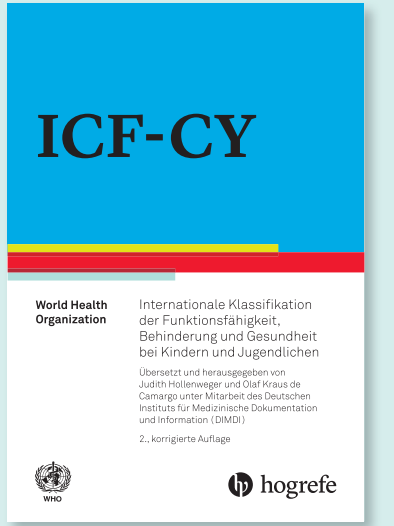

WHO - World Health Organization

\section{ICF-CY}

Internationale Klassifikation der Funktionsfähigkeit,

Behinderung und Gesundheit bei Kindern und Jugendlichen

Deutsche Ausgabe herausgegeben von Judith Hollenweger / Olaf Kraus de Camargo.

Übersetzt von Judith Hollenweger / Olaf Kraus de Camargo.

2., korrigierte Auflage 2017.320 S., Kt

$€ 39,95 / \mathrm{CHF} 48.50$

ISBN 978-3-456-85812-8

Mit der ICF hat die WHO die betroffene Person und nicht ihre Symptome ins Zentrum gestellt und damit eine einheitliche Sprache geschaffen, die aus Komponenten der Kör- perfunktionen und -strukturen, der möglichen Aktivitäten und der sozialen Teilhabe sowie der relevanten Umgebungsfaktoren besteht. 